and 2-10 years, the parents or the head of household were interviewed to obtain the responses. Impairments, activity limitations and restriction of participation were considered in defining disability which is consistent with the International Classification of Functioning, Disability and Health framework.

Results The overall age standardised prevalence of disability per 1000 was 46.5 (95\% confidence interval, 44.4-48.6). The prevalence was significantly high among the respondents living in rural areas at 50.2 (47.7-52.7), compared to urban areas at 31.0 (27.0-35.0). Overall, females had more disability 50.0 (46.953.1) than males 43.4 (40.5-46.3). Educational deprivation was closely linked to higher prevalence of disability. Commonly reported prevalence (per 1000) of cause specific disability was illness (20.2) followed by congenital (9.4) and injury (6.8) and these were consistent in males and females.

Conclusions Disability is a common problem in this typical district of Bangladesh, which is largely generalizable. Interventions at community level with special attention to the socio-economically deprived group are warranted.

\section{UNDERSTANDING TRAJECTORIES OF MENTAL DISTRESS AFTER MODERATE OR SEVERE INJURY AMONG ADULTS IN KENYA}

${ }^{1}$ Yuen W Hung, ${ }^{1}$ Abdulgafoor M Bachani, ${ }^{2}$ Stephanie Aketch, ${ }^{2}$ Ryan Duly, 'Wietse Tol, ${ }^{2}$ Kent A Stevens, 'Adnan A Hyder. ${ }^{1} J o h n s$ Hopkins University Bloomberg School of Public Health, USA; ${ }^{2}$ Handicap International, Kenya

\subsection{6/injuryprev-2016-042156.115}

Background Injuries present a major burden to populations in developing countries. Despite major psychological distress have been found among injury survivors in developed settings, there has been a lack of literature on the mental health consequences among all types of injury in developing countries, especially in sub-Saharan Africa.

Methods The Health, Economic and Long-term Social Impact of Injuries (HEALS) Study is a multi-country prospective longitudinal study currently underway. In Kenya, the study includes adult patients age 18 years or above who are hospitalised due to injury for at least one day in Kenyatta National Hospital. Eligible patients are being enrolled in the study until the sample size of 1000 patients is reached. Patients are interviewed in-person while in hospital, and followed up at 1, 2, and 4 months after hospital discharge through phone interviews. Distress symptoms severity is assessed using Hopkins Symptoms Checklist (HSCL-25) at baseline and follow-ups, and post-traumatic stress disorder symptoms are assessed at follow-up interviews using PTSD Checklist for DSM-5 (PCL-5).

Results 320 patients have enrolled in the study and completed baseline interview, 246 patients completed first follow-up interview, and 119 respondents completed second follow-up interview. Preliminary total score of HSCL-25 is 1.18 (SD: 0.17) at baseline, 1.15 (SD: 0.18) at first follow-up, and 1.10 (SD: 0.14) at second follow-up interview. Exploratory factor analysis will be conducted to determine the underlying factor of distress. Latent growth mixture modelling will be to determine the latent construct of mental distress. Risk factors such as sex, age, type of injury, severity of injury, and previous exposure to traumatic experience will be assessed.

Conclusions Mental distress continues months after hospitalisation among some injury patients. This highlights the importance of screening and attending to the mental health of patients in recovery from their injury.

\section{FUNCTION, HEALTH RELATED QUALITY OF LIFE AND COST AFTER INJURY IN A CITY OF NORTH INDIA: A MULTI SITE COHORT STUDY}

${ }^{1}$ Jagnoor Jagnoor, ${ }^{2}$ Shankar Prinja, ${ }^{3}$ Belinda Gabbe, ${ }^{1}$ Rebecca Q Ivers. ${ }^{1}$ University of Sydney, Australia; ${ }^{2}$ Post Graduate Institute for Medical Health, India; ${ }^{3}$ Monash University, Australia

\subsection{6/injuryprev-2016-042156.116}

Background The burden of traumatic injury in India is high, but remains ill-defined and poorly quantified. The aim of this project was to measure the impact of traumatic injuries on functioning and health-related quality-of-life (HRQoL), economic impactand to identify predictors of poor outcomes post-injury.

Methods A prospective observational study was conducted at three hospital sites in Chandigarh, North India in 2014-2015 for all ages admitted with an injury. Consent was sought and participants were followed at 1, 2, 4 and 12 months after injury; face to face or telephonic interviews collected data on socio-demographics, circumstances of injury, cost associated with injury, disability, function and health related quality of life. Interim analysis for 4 months is reported below, with 12 months interviews underway.

Results 2950 (90\% of eligible) participants were recruited, with a follow-up rate of $74 \%(2180)$ at 4 months. Road traffic injuries (1622/55\%) followed by falls (914/31\%) and burns (383/13\%) were the leading cause of injury; $86 \%$ were males, $79.5 \%$ were in paid employment at the time of injury. The average out of pocket expenditure per hospitalisation and up to 4 months post discharge was USD 388 (95\% CI: 332-441) and USD 946 (95\% CI: 771-1021) respectively. The prevalence of catastrophic expenditure was 30\% (95\% CI: 26.95-31.05), which was significantly associated with lower income quartile (OR 23.3 [95\% CI: 5.773.9]; p < 0.01), inpatient stay greater than 7 days (OR 8.8 [95\% CI: 3.8-20.6); p < 0.01), major surgery (OR 4.9 [95\% CI: $2.7-8.4] ; \mathrm{p}<0.01$ ), and occupation as wage labourers (OR 8.1 [95\% CI: 1.6-24.6]; $\mathrm{p}=0.01$ ).

Conclusions Injury has a substantial impact with a high proportion of patients sustaining catastrophic health expenditure, particularly the poor. Measures aimed at increasing public health spending for sustained prevention programs and providing financial risk protection to those injured deserve urgent priority in India.

\section{LONG-TERM HEALTH, ECONOMIC, AND SOCIAL IMPACT OF INJURY IN FOUR LOW- AND MIDDLE-INCOME COUNTRIES}

'Abdulgafoor M Bachani, 'Xiaoge Julia Zhang, 'Yuen Wai Hung, 'Casey Risko, ${ }^{1}$ Rantimi Olumasegun, ${ }^{2}$ Cuong Pham, ${ }^{2}$ La Ngoc Quang, ${ }^{3}$ Muhammad Fadhli Yusoff,


International Injury Research Unit, Johns Hopkins University Bloomberg School of Public Health, USA; ${ }^{2}$ Hanoi School of Public Health, Vietnam; ${ }^{3}$ nstitute Kesihatan Umum, Malaysia; ${ }^{4}$ Handicap International, Cambodia; ${ }^{5}$ Handicap International, Kenya

\subsection{6/injuryprev-2016-042156.117}

Background With $90 \%$ of the burden of injuries concentrated in low- and middle-income countries (LMICs), the impact on individuals, families, and society, especially in the case of non-fatal injuries, is exacerbated by the absence of insurance or social support mechanisms. There is a dearth of information in the literature on the occurrence of non-fatal injuries, and their long-term consequences. This study aims to understand the health (disability), social and economic impact of injuries in LMICs. 
Methods This is a prospective cohort study of 4200 moderate to severely injured patients at seven government hospitals in four LMICs (Cambodia, Kenya, Malaysia, and Vietnam). We administer a baseline and four follow-up surveys at home (at 1, 2, 4, and 12 months after discharge) to participants about their injury, functioning and disability, medical costs, employment, household composition and roles, as well as support systems (social \& economic).

Results The on-going study has enrolled 2293 individuals at the seven hospitals (Cambodia: 595, Kenya: 320, Malaysia: 284, and Vietnam: 1094). Participants are $72-84 \%$ male, with an average age of 30-42 years. The top cause of injury across all countries is road traffic injury $(47-72 \%)$. The second leading cause is falls (17-20\%), except for Kenya, where assaults rank second (20\%) and falls rank third (16\%). Changes in levels of disability, health care costs, productivity, household economic status, and roles of family members, as well as the respective influencing factors will be examined using marginal models with Generalised Estimating Equations (GEE) approach.

Conclusions Injury predominantly affects young males at their prime, having a significant impact not only on themselves, but their family as well as the society. This study will lead to a better understanding of the far reaching health, social, and economic impact of injuries. Data collected could be used to guide policy and programs in each of the implementing countries.

\section{FINANCIAL BURDEN OF INJURY AMONG A PROSPECTIVE COHORT OF INJURY PATIENTS IN MALAYSIA}

${ }^{1}$ Abdulgafoor M Bachani, ${ }^{1}$ Xiaoge Julia Zhang, ${ }^{2}$ Muhammad Fadhli Mohd Yusoff, ${ }^{2}$ Hamizatul Akmal binti Abd Hamid bin, ${ }^{2}$ Mohamad Naim Mohamad Rasidi, ${ }^{1}$ Adnan A Hyder. 'Johns Hopkins International Injury Research Unit, Johns Hopkins University Bloomberg School of Public Health, USA; ${ }^{2}$ Institute for Public Health, Malaysia Ministry of Health, Malaysia

\subsection{6/injuryprev-2016-042156.118}

Background Malaysia is rapidly developing and facing an increase in the burden of injuries, which disproportionally affect young and economically productive individuals. This study aims to understand injury-related economic burden on individuals and their families over time.

Methods We are recruiting a prospective cohort of 1200 moderately to severely injured patients from two government hospitals in Selangor and Kedah, Malaysia. We administer a baseline and four follow-up surveys $(1,2,4,12$ months after discharge) to subjects about socio-demographic characteristics, injury, family structure, and income changes. We obtain medical costs (direct costs) from medical bills and expenditure diaries. The outcome measures are direct and indirect costs.

Results Of 284 participants enrolled, $75 \%$ are male and $82 \%$ are Malay. Road traffic injury (70\%), fall (17\%) and blunt object injury $(17 \%)$ are the top three causes of injuries. On average, participants were hospitalised for 9.7 days (SD: 11.8), which was associated with productivity losses equivalent to RM411 (SD: 600) per person. Before injury, the average monthly income of participants was RM1304 (SD: 940), and the average medical care costs were RM156 (SD: 152). Income during hospitalisation was reduced in $24 \%$ of participants (RM534 on average). We will use marginal models with Generalised Estimating Equations to examine the trend of average costs. We will estimate average income changes by modelling the likelihood of having income reduction using logistic regression and size of income reduction using generalised linear regression.

Conclusions Injury brings about significant financial stress to individuals through direct medical costs, productivity loss and income reduction. This study will provide empirical evidence on the trend of injury costs and identify factors associated with high injury costs in Malaysia.

\section{Behavioural Science Research Informs Interventions to Reduce Child Injury - Panel Discussion}

\section{CLUSTER-RANDOMISED CONTROLLED TRIAL OF A FIRE SAFETY INJURY PREVENTION BRIEFING IN CHILDREN'S CENTRES}

${ }^{1}$ Toity Deave, ${ }^{2}$ Adrian Hawkins, ${ }^{3}$ Mike Hayes, ${ }^{4}$ Nicola Cooper, ${ }^{5}$ Carol Coupland ${ }^{6}$ Gosia Majsak-Newman, ${ }^{1}$ Trudy Goodenough, ${ }^{2}$ Elaine McColl, ${ }^{7}$ Richard Reading, ${ }^{5}$ Denise Kendrick. ${ }^{1}$ University of the West of England Bristol, UK; ${ }^{2}$ Newcastle University, Newcastle Upon Tyne, UK; ${ }^{3}$ Child Accident Prevention Trust, London, UK; ${ }^{4}$ University of Leicester, Leicester UK; ${ }^{5}$ University of Nottingham, Nottingham, UK; ${ }^{6}$ South Norfolk CCG, Norwich, UK; ${ }^{7}$ Norfolk and Norwich University Hospital, Norwich, UK

\subsection{6/injuryprev-2016-042156.119}

Background The UK has high mortality rates for fire-related deaths in children aged $0-14$ years with steep social gradients. Interventions to promote fire safety practices can be effective in reducing the risk of fire-related injury. We therefore developed an evidence-based fire safety intervention comprising an Injury Prevention Briefing (IPB), training and facilitation for use in children's centres.

Methods A cluster randomised controlled trial, with integrated qualitative study, was conducted across four study sites in England involving children's centres in disadvantaged areas; participants were staff and families attending those centres. Centres were stratified by study site and randomised within strata to one of three arms: IPB plus facilitation (IPB + ), IPB only, usual care. $\mathrm{IPB}+$ centres received initial training and facilitation at months 1, 3, and 8. Data collected comprised baseline and 12 months parent- and staff-completed questionnaires, home safety activity logs and staff interviews. The primary outcome was the proportion of families with a home fire-escape plan. Treatment arms were compared by using multilevel models to account for clustering by centre.

Results 1112 parents at 36 CCs participated. There was no significant effect of the intervention on family possession of fireescape plans (AOR IPB only vs. usual care: 0.93, 95\% CI: 0.58, 1.49; AOR IPB+ vs. usual care 1.41, 95\% CI: 0.91, 2.20). However, significantly more families in the intervention arms achieved more fire-escape planning behaviours (AOR IPB only vs. usual care: $2.56,95 \%$ CI: 01.38, 4.76; AOR IPB+ vs. usual care 1.78 , 95\% CI: 1.01, 3.15).

Conclusions Our study demonstrated that children's centres can deliver an injury prevention intervention to families in disadvantaged communities and achieve changes in home safety behaviours. 